Review Article

\title{
Can Vascular Endothelial Growth Factor and Microvessel Density Be Used as Prognostic Biomarkers for Colorectal Cancer? A Systematic Review and Meta-Analysis
}

\author{
Yibaina Wang, Xiaoping Yao, Jie Ge, Fulan Hu, and Yashuang Zhao \\ Department of Epidemiology, Public Health College, Harbin Medical University, 157 Baojian Street, \\ Nangang District, Harbin, Heilongjiang 150081, China \\ Correspondence should be addressed to Yashuang Zhao; zhao_yashuang@263.net
}

Received 11 November 2013; Accepted 29 January 2014; Published 27 March 2014

Academic Editors: E. Z. Dajani and C. Tosetti

Copyright (C) 2014 Yibaina Wang et al. This is an open access article distributed under the Creative Commons Attribution License, which permits unrestricted use, distribution, and reproduction in any medium, provided the original work is properly cited.

\begin{abstract}
Background. Vascular endothelial growth factor (VEGF) and microvessel density (MVD) are associated with greater incidence of metastases and decreased survival. Whether they can be used as prognostic indicators of colorectal cancer (CRC) is still controversial. Methods. The authors performed a meta-analysis using the results of a literature search of databases of PubMed and EMBASE, and the references of articles included in the analysis. Meta-analysis was performed using random effects model and hazard ratios (HRs) and 95\% confidence intervals (CIs) as effect measures. Results. Twenty studies contributed to the analysis of VEGF, of which 16 were used for overall survival (OS) and 9 for disease-free survival (DFS). High VEGF levels has a relationship with unfavorable survival (OS: $\mathrm{HR}=1.98,95 \% \mathrm{CI}: 1.30-3.02$; DFS: $\mathrm{HR}=2.10,95 \% \mathrm{CI}: 1.26-3.49$ ) and a 4.22 -fold increase in the rate of distant metastases. Analysis was performed on 18 studies for MVD; the results showed that patients with high MVD expression in tumors appeared to have poorer overall survival ( $\mathrm{HR}=1.39,95 \% \mathrm{CI}: 1.22-1.58)$ and were at a greater risk of having unfavorable clinical characteristics related to prognosis. Corresponding results were obtained from quantitative and/or qualitative analysis of clinicopathological. Conclusions. The meta-analysis demonstrates that VEGF and MVD can be used as prognostic biomarkers for CRC patients.
\end{abstract}

\section{Introduction}

Neoangiogenesis, the formation of new blood vessels from existing ones, plays an important role in the growth and progression of tumors [1]. As a tumor grows, the lack of a sufficient blood supply creates a hypoxic environment that stimulates the release of factors such as hypoxia inducible factor- (HIF-) $1 \alpha$ to induce angiogenesis by activating the transcription of vascular endothelial growth factor (VEGF) $[2,3]$. VEGF is an endothelial, cell-specific mitogen and an angiogenic inducer, as well as a mediator of vascular permeability that was first recognized by Leung et al. in 1989 [4]. Along with fibroblast growth factors, transforming growth factors, tumor necrosis factor, interleukin-8, and various angiopoietins, VEGFs are potent inducers of the angiogenic switch. This switch is characterized by a sequence of steps beginning with vessel dilation and the detachment of pericytes from preexisting vessels followed by angiogenic sprouting and the proliferation of endothelial cells, new vessel formation, and recruitment of perivascular cells [5]. The use of microvessel density (MVD) as a surrogate marker for tumor angiogenesis has also been reported.

Bevacizumab is a specific anti-VEGF drug that has recently attracted the attention of clinical oncologists. A series of multicenter, randomized controlled clinical trials have evaluated the efficacy and safety of bevacizumab when used in the survival of patients with metastatic colorectal cancer (CRC) [6-9].

A meta-analysis including 10 VEGF studies and 18 MVD studies was conducted by be G. Des Guetz 2006; this metaanalysis suggested that the expression of both VEGF and MVD significantly associated with poor overall survival (OS) for colorectal cancer [10]. However, the study measured the effect by using relative risk, which only measures the number 
of events and does not illustrate the time they occur. Hazard ratios (HRs) take into account the number and timing of events, but the time until last follow-up for each patient who has not experienced an event is censored [11]. Since 2006, several additional studies using larger sample sizes and multivariate Cox analyses have been reported. The authors conducted a systematic review of these new data by performing a meta-analysis, which for the first time used pooled HR data to evaluate whether VEGF or MVD expression was associated with prognosis in CRC.

\section{Materials and Methods}

2.1. Search Strategy and Selection Criteria. A comprehensive, systematic literature review was performed independently by Y.B.N.W. and X.P.Y. from the inception to February 30, 2013, using PubMed, EMBASE, and the Cochrane database to identify potentially relevant studies. The structured search was based on four main medical subject headings and keywords: microvessel density, VEGF, colorectal cancer, and prognosis.

Based on careful reading of the abstracts, studies were selected for further analysis. The authors manually reviewed all of the reference lists for the selected studies to identify additional articles for inclusion. This cross-referencing strategy was performed until no further eligible publications were identified.

To be included, the studies had to meet the following criteria: the original study had to be designed to assess the association between the expression of VEGF or MVD and the prognosis of CRC patients, and the study had to provide primary outcomes on survival, such as HRs for Cox proportional hazard model. Only English language studies were included.

To avoid overlapping data in duplicate publications, we analyzed all of the author names for each study, the different medical institutions involved, and the timeframe of the research. In the case where multiple studies using the same patient population were identified, the combined data were included. We then adapted a validity questionnaire to weight the quality and applicability of the studies included (see Online Resource 1 in the Supplementary Material available online at http://dx.doi.org/10.1155/2014/102736) [12].

2.2. Data Extraction. Two reviewers independently extracted the relevant data from the full texts of all included studies according to a predefined protocol. The standardized dataextraction form consisted of the following items: first author, year of publication, characteristics of the study population, duration of follow-up, counting method for VEGF and MVD, cut-off values for VEGF and MVD, HRs and their 95\% confidence intervals (CIs) on OS, and/or disease-free survival (DFS) between the high and low groups in multivariate analysis. Differences in extracted data were crosschecked until consensus was reached.

2.3. Statistical Analysis. Pooled HRs and their 95\% CIs on OS and/or DFS between the high and low groups were calculated using the maximum adjusted HR for each included study. To estimate the effect of VEGF or MVD on the prognosis of the CRC patients, the parameters were considered or transformed as binary variables using the cut-off value for the parameter (usually the median) [13]. The heterogeneity among studies in the meta-analysis was evaluated by $I^{2}$ statistics and found to be $I^{2}>50 \%$; as this was considered to be unacceptable, a random effects model was adopted [14]. To assess the influence of publication year, the authors conducted a cumulative meta-analysis in which they accumulated the included studies chronologically by year of publication. Sensitivity analysis was performed by comparing the combined results before and after one study was sequentially removed from the meta-analysis. Subgroup analyses were conducted by age, geographic area, and duration of followup to evaluate all conceivable sources of heterogeneity. The authors also evaluated the pooled association between VEGF and MVD expression and different clinical characteristics, both qualitatively and quantitatively. Additionally, the papers used funnel plots to detect publication bias and further qualitative bias using Egger's regression test and Begg's rank correlation test [15]. If bias was present, Duval and Tweedie's trim-and-fill method was adopted to adjust the original pooled result. All statistical analyses were performed using Comprehensive Meta-Analysis version 2.0.

\section{Results and Discussion}

3.1. Literature Search and Description of Included Studies. Figure 1 shows the flow diagram for the selection of included studies. The systematic literatures search yielded 1,143 total articles, 1,083 of which were excluded either because of a lack of survival data or an overlap with other studies. Sixty references were used in the analysis of association between VEGF/MVD expression and clinical and pathology feature; 34 articles met the inclusion criteria and were included in the final meta-analysis. Online Resources 2 and 3 summarize the baseline characteristics and quality scoring of these studies.

The final 34 studies represented 3,618 patients and ranged in sample size from 31 to 278 (median, 101 patients). Three studies $[1,16,17]$ included primary CRC only, 6 studies [1823] focused only on advanced patients, and the remaining studies examined patients at all stages of disease.

VEGF and MVD expression were assessed in CRC specimens excised before treatment with either chemotherapy or preoperative radiotherapy, except in four studies $[18,19,22$, 24].

The methodological quality scores reflect the high quality of the included studies; only 7 studies were determined to be level $c$, meaning they were imperfect in the research design, lab methodology, or statistical analysis.

3.2. VEGF Expression and Prognosis for CRC. Based on the study data available for evaluating the association between VEGF expression and prognosis, the pooled HR was 1.98 (95\% CI: $1.30-3.02 ; P<0.01 ; I^{2}=73.64$ ) for overall survival $[1,16,18,24-36]$ and 2.10 (95\% CI: 1.26-3.49; $P<0.01$; 


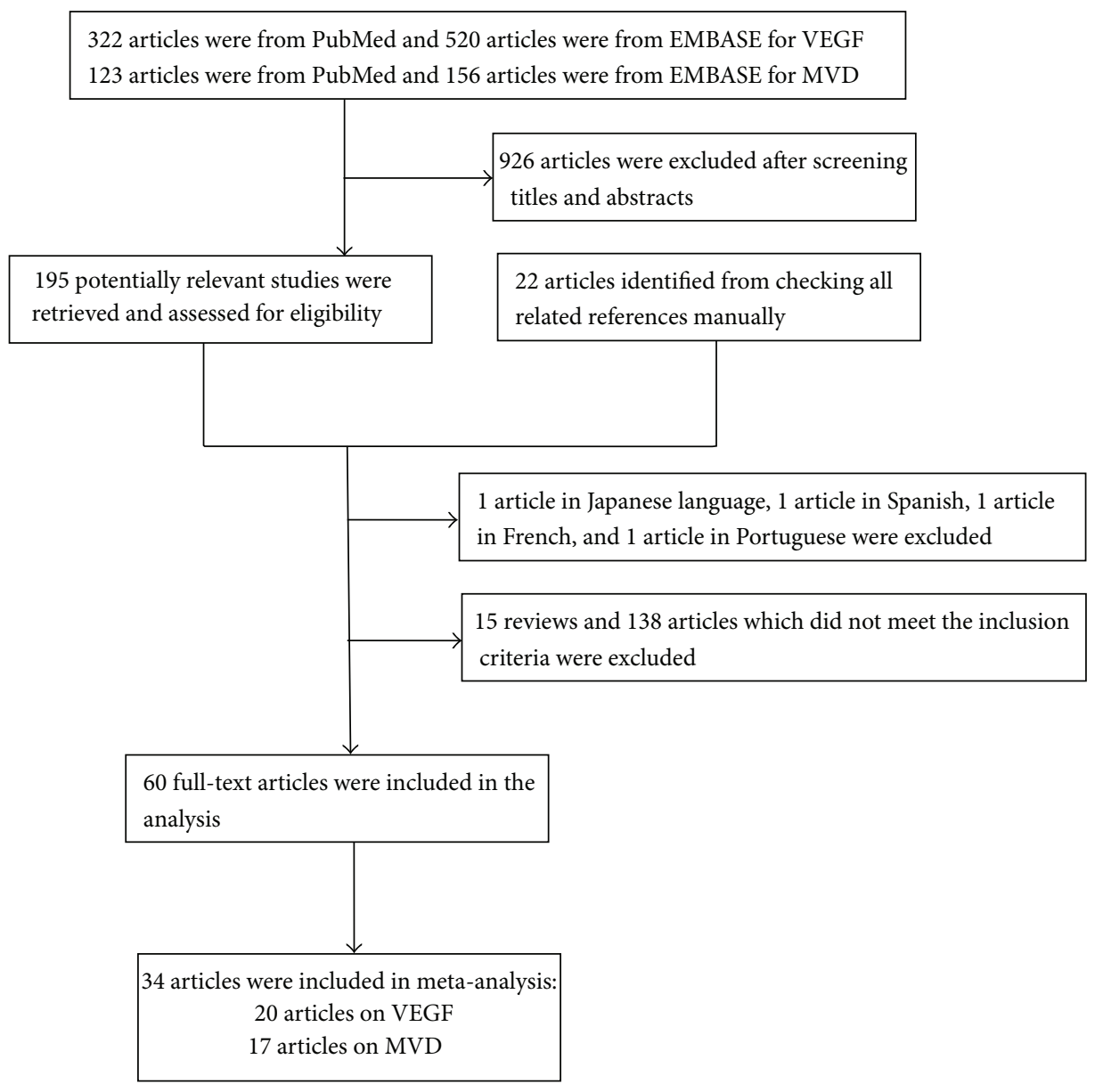

FIgURE 1: The flow diagram of the studies search.

$\left.I^{2}=71.44\right)$ for disease-free survival $[18,19,26,27,31,35,37-$ 39] based on the random effects model (Figures 2 and 3 ). The cumulative meta-analysis showed that the strength of this association varied by approximately 2.00. However, the variant trended small, and the pooled result approached stability with time (Figures 4 and 5). Further subgroup analyses were carried out based on age at diagnosis, nationality, and the duration of follow-up. VEGF expression has a relationship with OS both in the elderly group (pooled HR $=2.18$, 95\% CI: 1.11-4.30; $P=0.02$ ) and the nonelderly group (pooled $\mathrm{HR}=$ 1.91, 95\% CI: 1.05-3.46; $P=0.03$ ) ( $P=0.77$ for comparison). The prognostic effect was more apparent in those of European ancestry (pooled HR $=3.42$, 95\% CI: 1.90-6.14; $P<0.01$ ) than among Asians and Americans $(P<0.01)$. Additionally, the association between VEGF and survival was stronger in the four studies with a median follow-up time longer than 60 months (pooled HR $=3.90,95 \% \mathrm{CI}: 2.15-7.07 ; P<0.01$ ) than in studies with a follow-up period shorter than 60 months (pooled HR $=1.39,95 \%$ CI: $0.81-2.36 ; P=0.23)(P=0.01$ for comparison) (Table 1).

The associations between VEGF expression and clinical features were provided by 19 studies $[1,19,29-33,40-51]$ (Table 2). Notably, higher rates of VEGF expression were significantly associated with the following clinicopathological features: lymph node metastasis (pooled OR $=2.51,95 \% \mathrm{CI}$ : $1.51-4.15 ; P<0.01$ ) and vascular metastasis (pooled $\mathrm{OR}=$ 2.38, 95\% CI: 1.49-3.79; $P<0.01)$. Similarly, the incidence of tumor distant metastases tended to be higher in patients with high rather than low expression of VEGF (pooled OR $=4.22$, 95\% CI: 2.93-6.06; $P<0.01)$.

3.3. MVD Expression and Prognosis for CRC. The MVD meta-analysis using 18 studies $[1,3,16,17,21-23,27,52-$ 60] showed that MVD was associated with overall survival with a pooled HR of 1.39 (95\% CI: 1.22-1.58; $P<0.01$; $I^{2}=83.14$ ) (Figure 6). Additionally, the cumulative metaanalysis demonstrated a decreasing association over time that eventually tended to be statistically significant and stable (Figure 7). Subgroup analysis found that the significant associations remained in the nonelderly patients (pooled $\mathrm{HR}=2.17,95 \% \mathrm{CI}: 1.23-3.83 ; P<0.01)$ but not in the elderly patients (pooled HR $=1.28,95 \%$ CI: 0.94-1.74; $P=0.11$ ). However, the difference between the two groups was not statistically significant $(P=0.11$ for comparison). The pooled HR in Asians (pooled HR = 1.35, 95\% CI: 1.12-1.63; $P<0.01$ ) was similar to that of Europeans (pooled HR $=1.62,95 \%$ CI: $1.24-2.12 ; P<0.01)$. Only two American reports studied the association between MVD and the prognosis of CRC, 


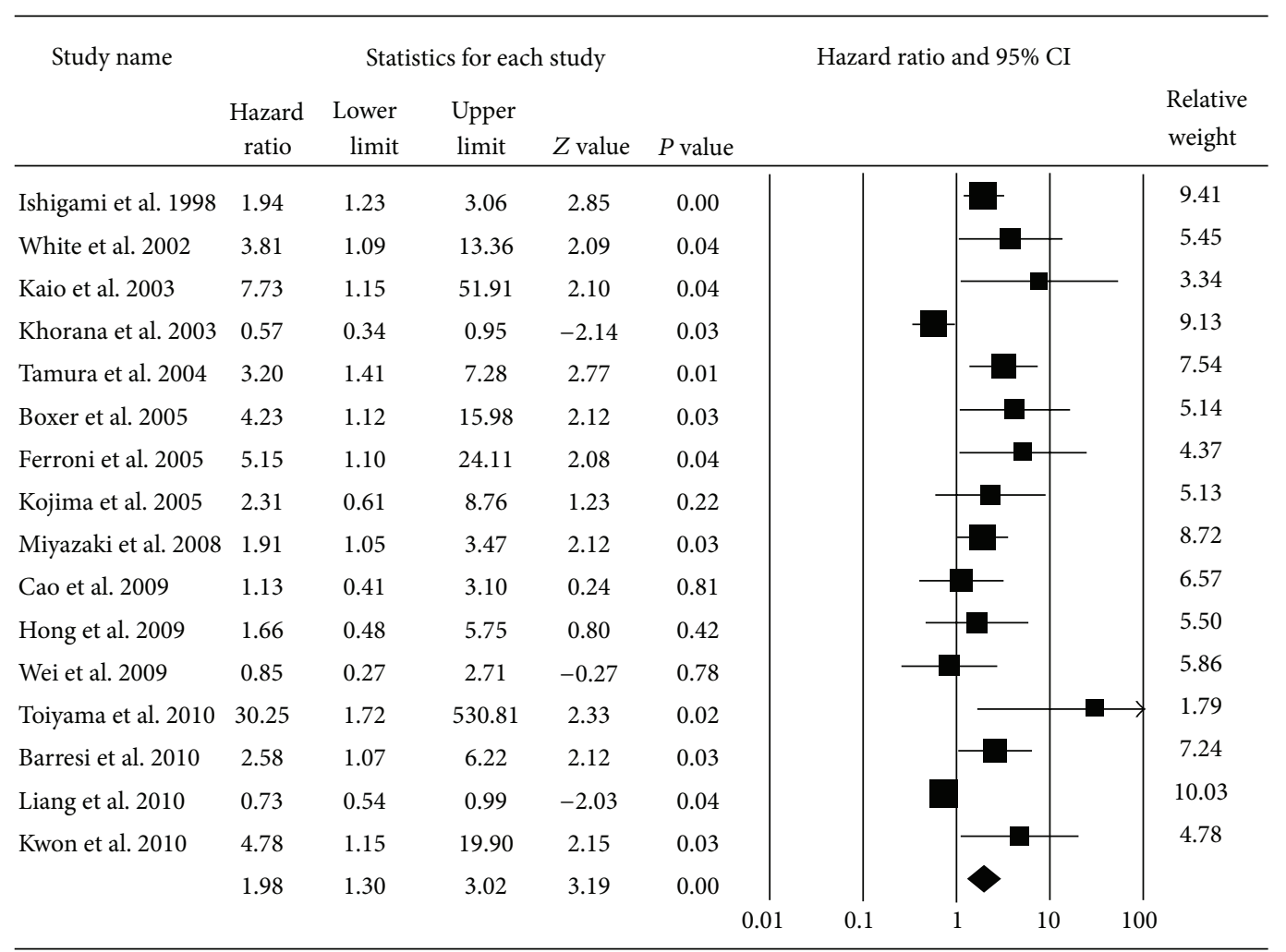

FIGURE 2: Forest plot for the association between VEGF expression and overall survival of CRC. The squares and horizontal lines represent the HRs and 95\% confidence intervals (CIs) in the individual studies; the size of each square represents the weighting assigned to the corresponding study. The diamond indicates the pooled HRs.

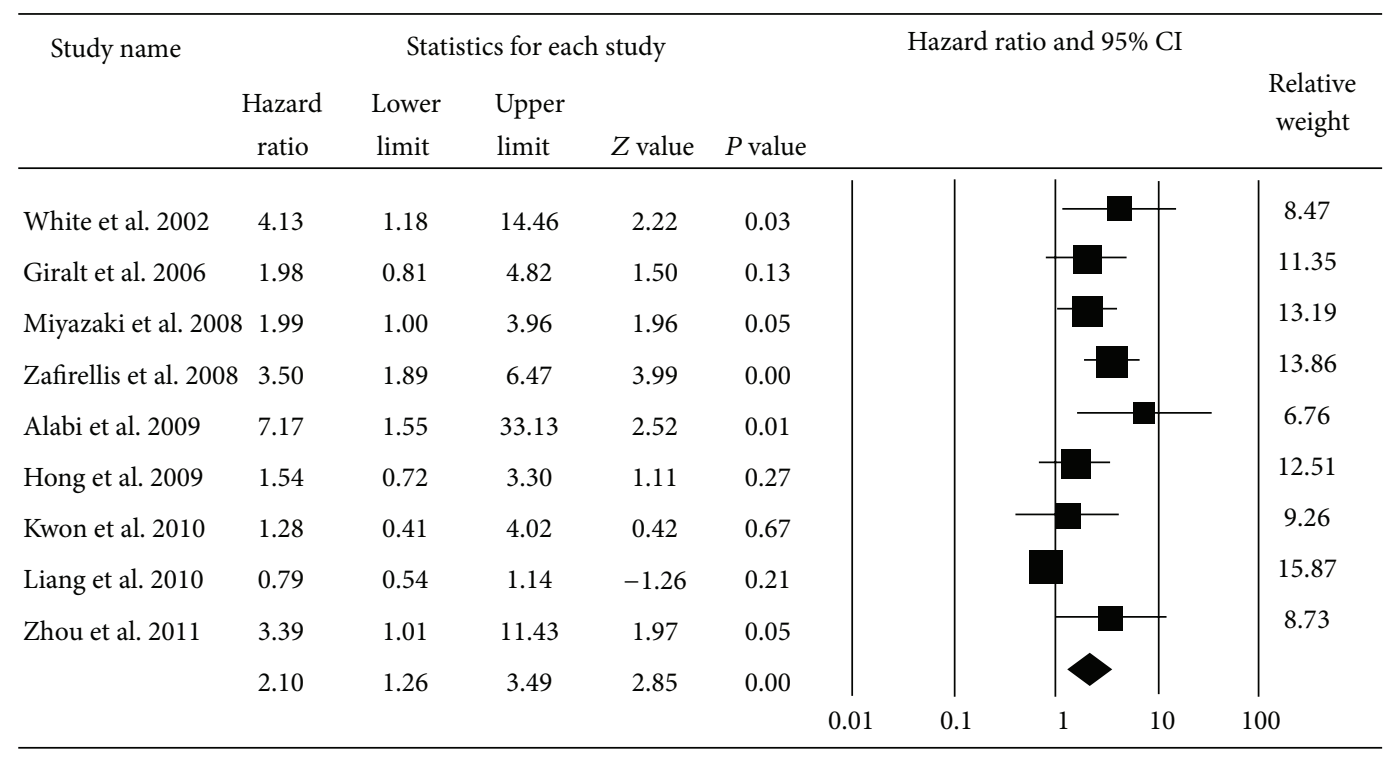

FIGURE 3: Forest plot for the association between VEGF expression and disease-free survival of CRC.

yielding a pooled HR of 1.57 (95\% CI: $0.50-4.95 ; P=0.44)$ ( $P=0.55$ for comparison). The authors also noted that different durations of follow-up did not directly affect the association between MVD and survival; the pooled HR for follow-up longer than 60 months (pooled $\mathrm{HR}=1.60,95 \%$ CI: 1.19-2.16; $P<0.01)$ was similar to the value for follow-up of less than 60 months (pooled HR = 1.35, 95\% CI: 1.14-1.60; $P<0.01)(P=0.33$ for comparison) (Table 1$)$.

Further examination of the associations between MVD and clinicopathological factors used 25 studies [1, 3, 16, $17,23,32,46,49,54,55,57,61-74]$. The analysis revealed a more pronounced blood vessel density value in patients 


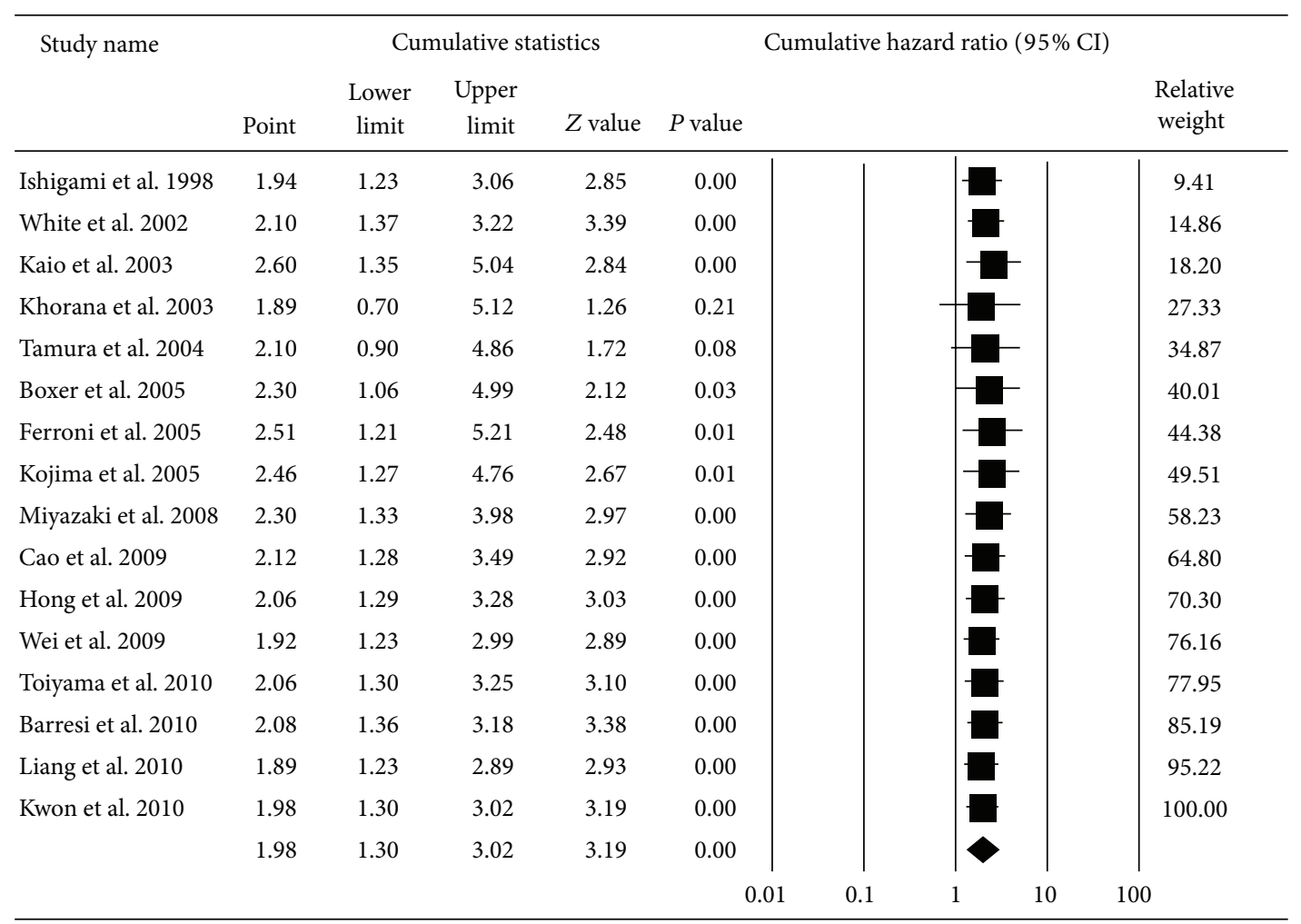

FIGURE 4: Cumulative meta-analysis for the association between VEGF expression and overall survival of CRC.

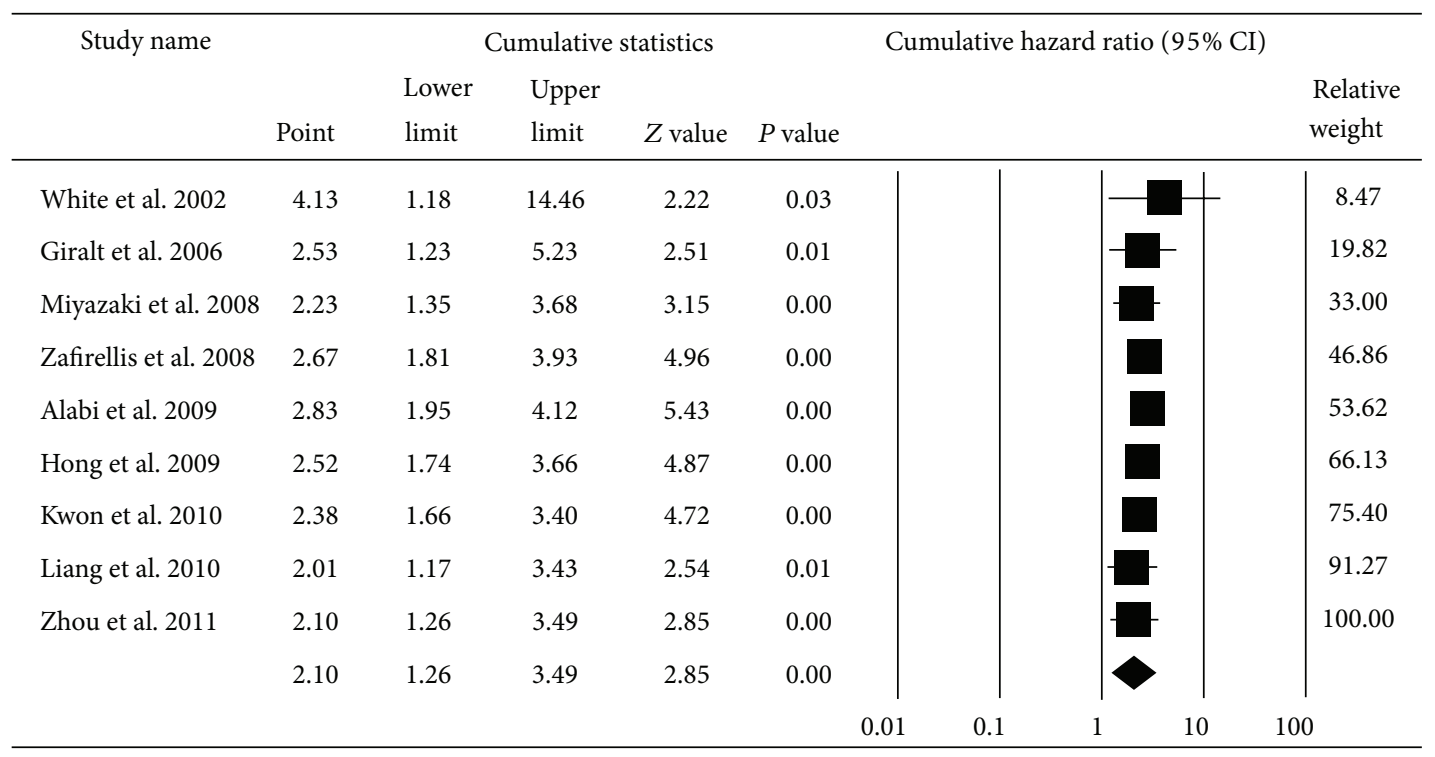

FIGURE 5: Cumulative meta-analysis for the association between VEGF expression and disease-free survival of CRC.

with vascular metastasis compared to those without (pooled $\mathrm{OR}=1.43,95 \%$ CI: 1.06-1.92; $P=0.02$; mean difference (MD): 13.99; standardized mean difference (SMD): 0.60) and in patients with lymph node metastasis compared to those without (pooled OR $=1.84,95 \%$ CI: 1.19-2.85; $P<0.01$; MD: 7.97; SMD: 0.45). Similarly, an increased incidence of distant metastases was seen in those with a high level of
MVD expression compared to those with low expression (MD: 13.14; 1.43) (Table 3).

3.4. Sensitivity Analysis. Based on studies of the association between VEGF and prognosis, each article had a balanced weight of less than $10 \%$. However, in 18 studies on MVD, 3 studies provided the largest weight in the analysis; further 


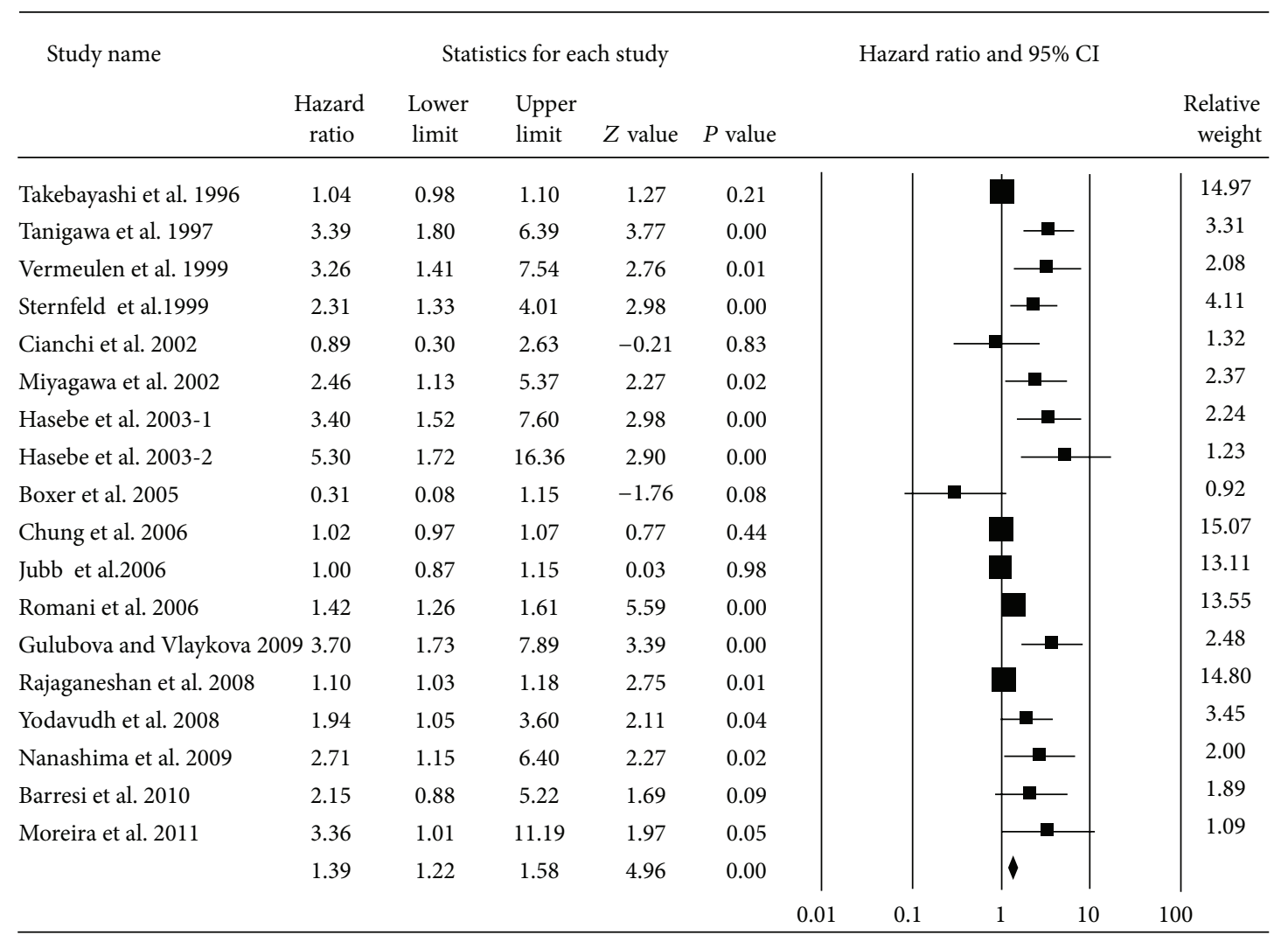

FIGURE 6: Forest plot for the association between MVD expression and overall survival of CRC.

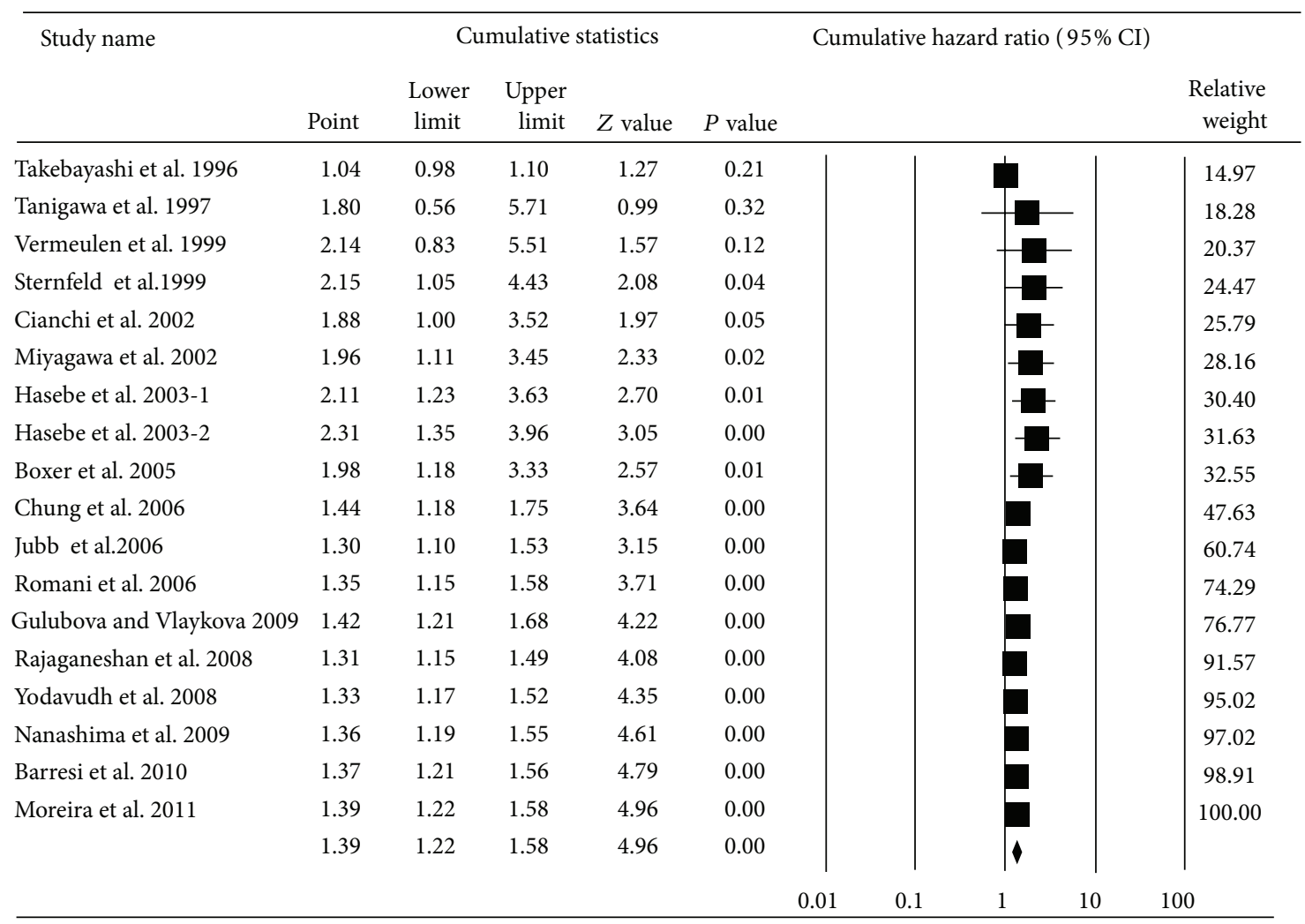

FIGURE 7: Cumulative meta-analysis for the association between MVD expression and overall survival of CRC. 


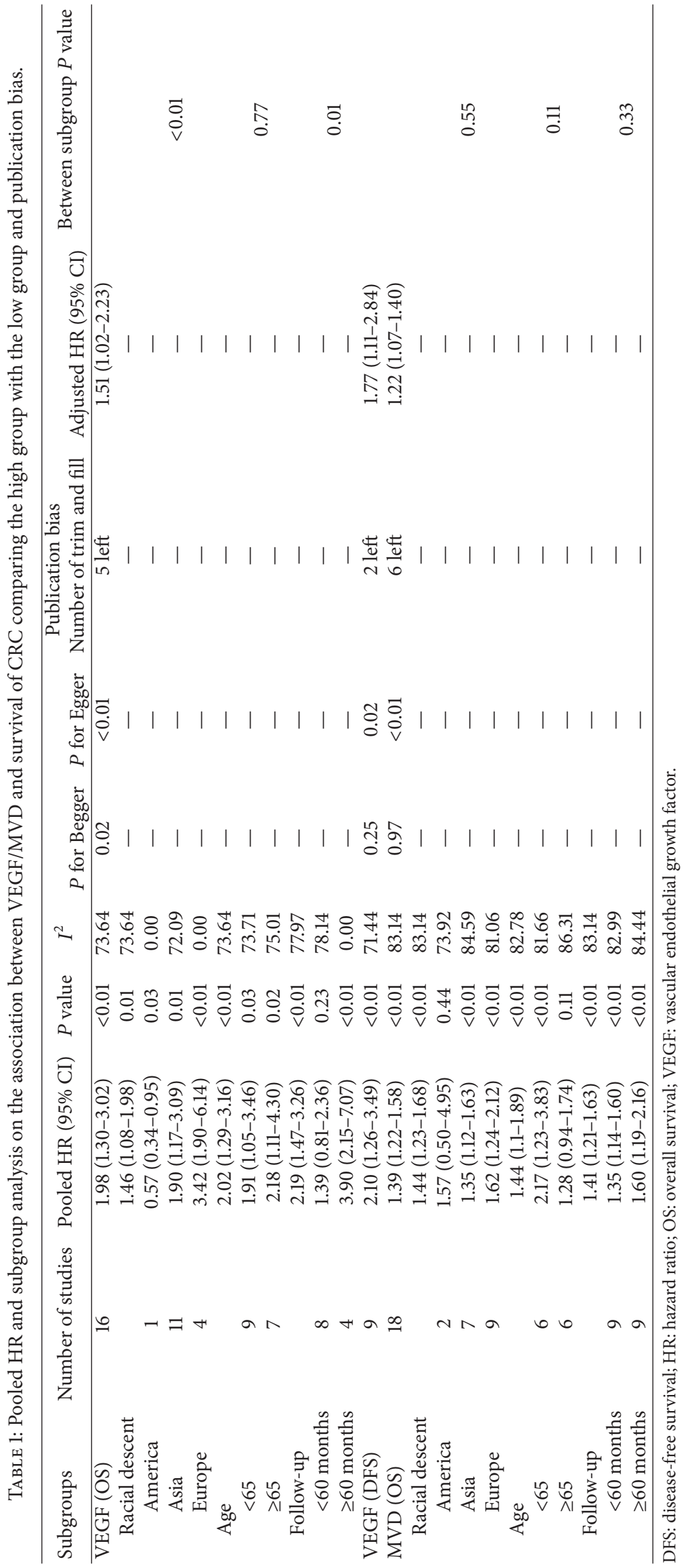


TABLE 2: Pooled ORs for the association between VEGF expression and clinical and pathology features.

\begin{tabular}{lccc}
\hline & $\begin{array}{c}\text { Number of } \\
\text { studies }\end{array}$ & $\begin{array}{c}\text { Pooled OR } \\
(95 \% \mathrm{CI})\end{array}$ & $P$ value \\
\hline Gender $^{1}$ & 13 & $1.08(0.84-1.38)$ & 0.55 \\
Distant metastasis $^{2}$ & 8 & $4.22(2.93-6.06)$ & $<0.01$ \\
Lymph node metastasis $^{2}$ & 15 & $2.51(1.51-4.15)$ & $<0.01$ \\
Lymph metastasis $^{2}$ & 7 & $1.57(0.97-2.54)$ & 0.06 \\
Vascular metastasis $^{2}$ & 9 & $2.38(1.49-3.79)$ & $<0.01$ \\
TNM $^{3}$ & 13 & $1.65(0.92-2.96)$ & 0.09 \\
\hline
\end{tabular}

OR: odds ratio; VEGF: vascular endothelial growth factor.

${ }^{1}$ Male versus female; ${ }^{2}$ metastasis of positive versus metastasis of negative; ${ }^{3}$ I and II versus III and IV.

sensitivity analysis after removing these studies by Rajaganeshan et al. [22], Chung et al. [21], and Takebayashi et al. [59] did not substantially impact the pooled results. When the analysis was restricted to studies where specimens were collected before chemotherapy, the pooled results for VEGF and MVD did not substantially change $\left(\right.$ pooled $\mathrm{OR}_{\mathrm{OS}-\mathrm{VEGF}}=$ 1.89, 95\% CI: 1.23-2.92; $P<0.01$; pooled $\mathrm{OR}_{\mathrm{DFS}-\mathrm{VEGF}}=2.13$, 95\% CI: $1.21-3.77 ; P<0.01$; pooled $\mathrm{OR}_{\mathrm{OS}-\mathrm{MVD}}=1.53,95 \%$ CI: $1.30-1.79 ; P<0.01)$.

3.5. Publication Bias. The presence of publication bias for the pooled association between VEGF expression and the prognosis of CRC was demonstrated visually using a funnel plot (Figure 8) and further qualitative analyses using Begg's rank correlation test or Egger's regression test. Additional analysis using Duval and Tweedie's trim-and-fill method found that the adjusted summary HRs did not change the results (HR $=1.68,95 \% \mathrm{CI}: 1.12-2.52)$.

The degree of asymmetry for the funnel plot (Figure 9) of the individual study results around the combined HR for OS between MVD expression and prognosis of CRC suggested that there was some degree of publication bias; this finding was confirmed by Egger's regression test $(P<0.05)$. Additional analysis was performed using Duval and Tweedie's trim-and-fill method, and it was found that the summary HRs $(\mathrm{HR}=1.27,95 \% \mathrm{CI}: 1.11-1.46)$ did not change the result, although the estimate of effect size tended to be small after adjustment.

\section{Discussion}

As the predominant angiogenesis factors in the growth and maturation of new vessels, VEGFs are associated with greater incidence of metastases and decreased survival. Results from previous studies assessing the relationship between VEGF, MVD, and the survival of CRC have been inconsistent. Until now, however, no meta-analysis has been conducted to evaluate the prognostic effects of VEGF/MVD using HR as an indicator, although a meta-analysis using OS and DFS was published by Des Guetz et al. 2006 [10]. It is known that HR is a more powerful factor than OS and DFS in survival data analysis [11]. The current systematic review and

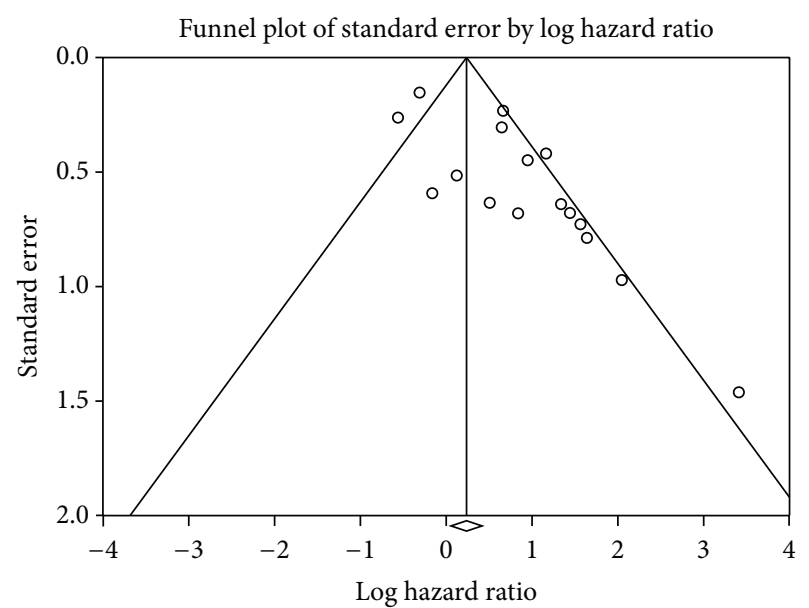

FIGURE 8: Publication bias pooled association between VEGF expression and overall survival.

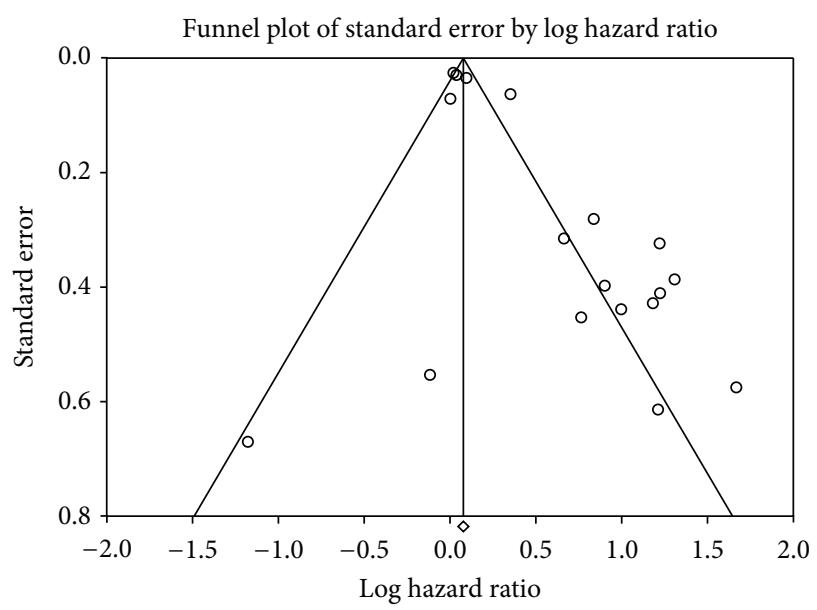

FIGURE 9: Publication bias pooled association between MVD expression and overall survival.

meta-analysis was therefore performed to determine whether the expression levels of VEGF and MVD in biopsy samples could accurately predict the prognosis of CRC patients. Our analysis, which pooled the maximum adjusted HRs from proportional hazard regression models, provided clear evidence that the expressions of VEGF and MVD were unfavorable prognostic predictors in colorectal cancer. Notably, overexpression of VEGF correlated with nearly two times the risk of death. Similarly, overexpression of MVD increased the risk of death in CRC patients by 39\%. Unfortunately, sufficient data on MVD and disease relapse could not be retrieved from existing studies. Because multivariate adjusted HRs avoid, as much as possible, confounding factors such as clinicopathological features, the authors concluded that both VEGF and MVD can independently provide valuable prognostic information for CRC patients, although the effect of MVD was weaker than VEGF.

Our data were consistent with the results of previous meta-analyses. The results from one meta-analysis of 51 
TABLE 3: Pooled ORs for the association between MVD expression and clinical and pathology features both in qualitative and quantitative analyses.

\begin{tabular}{|c|c|c|c|c|c|c|c|c|}
\hline & \multicolumn{3}{|c|}{ Qualitative analyses } & \multicolumn{5}{|c|}{ Quantitative analyses } \\
\hline & $\begin{array}{c}\text { Number } \\
\text { of } \\
\text { studies }\end{array}$ & $\begin{array}{c}\text { Pooled OR } \\
\text { (95\% CI) }\end{array}$ & $P$ value & $\begin{array}{c}\text { Number } \\
\text { of } \\
\text { studies }\end{array}$ & $\mathrm{MD}(95 \% \mathrm{CI})$ & $P$ value & SMD $(95 \% \mathrm{CI})$ & $P$ value \\
\hline Gender $^{1}$ & 8 & $0.94(0.71-1.24)$ & 0.66 & 11 & $-4.43(-18.82-9.96)$ & 0.55 & $-0.76(-1.36-0.15)$ & 0.01 \\
\hline Grade $^{2}$ & 11 & $1.47(0.86-2.49)$ & 0.15 & - & - & - & - & - \\
\hline Distant metastasis $^{3}$ & - & - & - & 8 & $13.14(-1.70-27.98)$ & 0.08 & $1.43(0.27-2.59)$ & 0.02 \\
\hline Lymph node metastasis ${ }^{3}$ & 8 & $1.84(1.19-2.85)$ & $<0.01$ & 10 & $7.97(3.64-12.31)$ & $<0.01$ & $0.45(0.22-0.69)$ & $<0.01$ \\
\hline Lymph metastasis $^{3}$ & 5 & $1.76(0.97-3.20)$ & 0.06 & 6 & $7.15(3.86-10.44)$ & $<0.01$ & $0.46(0.28-0.64)$ & $<0.01$ \\
\hline Vascular metastasis ${ }^{3}$ & 7 & $1.43(1.06-1.92)$ & 0.02 & 8 & $13.99(5.15-22.83)$ & $<0.01$ & $0.60(0.26-0.95)$ & $<0.01$ \\
\hline
\end{tabular}

MD: mean difference; MVD: microvessel density; OR: odds ratio; SMD: standardized mean difference.

${ }^{1}$ Male versus female, ${ }^{2}$ well and moderate versus poor and mucinous, and ${ }^{3}$ metastasis of positive versus metastasis of negative.

studies demonstrated that the overexpression of VEGF was significantly correlated with poor prognosis for patients with lung cancer [75]. Smith et al. [76] demonstrated that the expression of VEGF represented a significant and reproducible marker for adverse prognosis in resected pancreatic cancer as well. Similar results were also found in patients with hepatocellular cancer [77], breast cancer [13], and gastric cancer [78].

The papers further noticed that VEGF had a stronger association with overall survival of CRC in Europeans than in Asians. Our meta-analysis is the first to report this phenomenon. Further studies are needed to prove that the phenomenon exists and explore whether the difference is due to genetic susceptibility or other factors. When using age as a grouping factor, the effects of VEGF and MVD on prognosis are inconsistent, and the reasons for this remain unclear. The authors also observed that the duration of the followup period had an effect on the ability of VEGF and MVD to predict prognosis for CRC. The effect of VEGF or MVD as a significant prognostic indicator was much more obvious in those studies where the median follow-up period was longer than 60 months than in those with median follow-up periods shorter than 60 months.

In CRC patients, important prognostic parameters such as the TNM staging system, tumor grade, vascular-lymphatic invasion, and lymph node involvement have been identified. Thus, to understand the influence of VEGF and MVD expression on prognosis for CRC, we also assessed their relationship to clinicopathological variables. We observed that elevated VEGF apparently associated with more severe vascularlymphatic invasion and positive lymph node metastasis. Moreover, patients with high VEGF levels had a 4.22 -fold greater risk of developing distant metastases compared to patients with lower VEGF levels. Additionally, the analysis revealed that patients with increased MVD levels correlated with greater tumor aggressiveness, including poor differentiation and higher frequencies of vascular-lymphatic invasion, lymph node invasion, and distant metastases. The association between tumor angiogenesis and clinicopathologic factors thus provides further evidence that using VEGF and MVD as indicators for the prognosis of CRC is feasible.
Malignant tumors are crucially dependent on the process of angiogenesis. The key promoter of angiogenesis is VEGF, which may correlate with advanced clinical stage and worse prognosis [79]. The novel antiangiogenic agent, bevacizumab, is a recombinant, humanized monoclonal antibody that specifically blocks the activity of VEGF [80]. Large, randomized placebocontrolled trials $[8,9,81,82]$ and two pooled analyses $[83,84]$ have demonstrated that the addition of bevacizumab to traditional chemotherapy regimens provides statistically significant and clinically meaningful improvements in overall survival as well as response rate. However, little information about the relationship between VEGF status and survival outcomes in patients with CRC treated with bevacizumab was provided. Future studies will be needed to validate whether VEGF can be used as a biomarker to select patients who may benefit from bevacizumab and whether dose modification of bevacizumab may vary according to the degree of VEGF expression.

\section{Limitations}

Several limitations of this meta-analysis should be considered. First, the results should be interpreted in the context of treatment, but treatment was not uniform. Nonetheless, it is unlikely that the use of chemotherapy differed substantially based on the expression of VEGF or MVD. Thus, we concluded that the confounding effects of therapy would not have been substantial.

The next limitation was between-study heterogeneity. The data after subgroup analysis suggest that heterogeneity may be partly attributable to the grouped variable even though heterogeneity still existed. We believe that the observed discrepancy might be accounted for by the following: (1) the diversity across studies in patient selection, even though primary patients accounted for $47 \%$ of the total number in all studies; (2) the nonstandardized methodologies for assessing VEGF or MVD expression, such as the counting methods and whether or not assessors were blinded to the clinical data; and (3) the absence of uniform cut-off criteria for the studies (Online Resource 4); However, using the random effects 
model helped to diminish the effect of observed heterogeneity and led to results that were more conservative.

At last the meta-analysis was subject to publication bias; we attempted to overcome this limitation by identifying all of the relevant studies in key publications and adopting rigid inclusion criteria.

\section{Conclusion}

In conclusion, our systematic review and meta-analysis provided persuasive evidence that VEGF and MVD could be used as prognostic biomarkers for colorectal cancer. High expression levels of VEGF and MVD yielded poor prognosis results for patients after CRC surgery and indicated adverse effects on oncological clinical outcomes as demonstrated by the increased rates of vascular-lymphatic and lymph node metastasis and the increased incidence of distant metastases. The results can guide postoperative treatment of patients, especially the application of the bevacizumab.

\section{Conflict of Interests}

The authors declare that there is no conflict of interests regarding the publication of this paper.

\section{References}

[1] V. Barresi, C. di Gregorio, L. Regiani-Bonetti, M. Ponz-de Leon, G. Barresi, and E. Vitarelli, "Stage I colorectal carcinoma: VEGF immunohistochemical expression, microvessel density, and their correlation with clinical outcome," Virchows Archiv, vol. 457, no. 1, pp. 11-19, 2010.

[2] N. Ferrara, "VEGF and the quest for tumour angiogenesis factors," Nature Reviews Cancer, vol. 2, no. 10, pp. 795-803, 2002.

[3] S. Yodavudh, S. Tangjitgamol, and S. Puangsa-art, "Prognostic significance of microvessel density and mast cell density for the survival of Thai patients with primary colorectal cancer," Journal of the Medical Association of Thailand, vol. 91, no. 5, pp. 723-732, 2008.

[4] D. W. Leung, G. Cachianes, W.-J. Kuang, D. V. Goeddel, and N. Ferrara, "Vascular endothelial growth factor is a secreted angiogenic mitogen," Science, vol. 246, no. 4935, pp. 1306-1309, 1989.

[5] M. Guba, H. Seeliger, A. Kleespies, K.-W. Jauch, and C. Bruns, "Vascular endothelial growth factor in colorectal cancer," International Journal of Colorectal Disease, vol. 19, no. 6, pp. 510-517, 2004.

[6] H. Hurwitz, L. Fehrenbacher, W. Novotny et al., "Bevacizumab plus irinotecan, fluorouracil, and leucovorin for metastatic colorectal cancer," The New England Journal of Medicine, vol. 350, no. 23, pp. 2335-2342, 2004.

[7] L. B. Saltz, S. Clarke, E. Díaz-Rubio et al., "Bevacizumab in combination with oxaliplatin-based chemotherapy as first-line therapy in metastatic colorectal cancer: a randomized phase III study," Journal of Clinical Oncology, vol. 26, no. 12, pp. 20132019, 2008.

[8] B. J. Giantonio, P. J. Catalano, N. J. Meropol et al., "Bevacizumab in combination with oxaliplatin, fluorouracil, and leucovorin (FOLFOX4) for previously treated metastatic colorectal cancer: results from the Eastern Cooperative Oncology Group Study
E3200," Journal of Clinical Oncology, vol. 25, no. 12, pp. 15391544, 2007.

[9] F. F. Kabbinavar, J. Schulz, M. McCleod et al., "Addition of bevacizumab to bolus fluorouracil and leucovorin in first-line metastatic colorectal cancer: results of a randomized phase II trial," Journal of Clinical Oncology, vol. 23, no. 16, pp. 3697-3705, 2005.

[10] G. Des Guetz, B. Uzzan, P. Nicolas et al., "Microvessel density and VEGF expression are prognostic factors in colorectal cancer. Meta-analysis of the literature," British Journal of Cancer, vol. 94, no. 12, pp. 1823-1832, 2006.

[11] S. L. Spruance, J. E. Reid, M. Grace, and M. Samore, "Hazard ratio in clinical trials," Antimicrobial Agents and Chemotherapy, vol. 48, no. 8, pp. 2787-2792, 2004.

[12] E. Steels, M. Paesmans, T. Berghmans et al., "Role of p53 as a prognostic factor for survival in lung cancer: a systematic review of the literature with a meta-analysis," European Respiratory Journal, vol. 18, no. 4, pp. 705-719, 2001.

[13] B. Uzzan, P. Nicolas, M. Cucherat, and G.-Y. Perret, "Microvessel density as a prognostic factor in women with breast cancer: a systematic review of the literature and meta-analysis," Cancer Research, vol. 64, no. 9, pp. 2941-2955, 2004.

[14] R. DerSimonian and N. Laird, "Meta-analysis in clinical trials," Controlled Clinical Trials, vol. 7, no. 3, pp. 177-188, 1986.

[15] C. B. Begg and M. Mazumdar, "Operating characteristics of a rank correlation test for publication bias," Biometrics, vol. 50, no. 4, pp. 1088-1101, 1994.

[16] G. M. Boxer, E. Tsiompanou, T. Levine, R. Watson, and R. H. J. Begent, "Immunohistochemical expression of vascular endothelial growth factor and microvessel counting as prognostic indicators in node-negative colorectal cancer," Tumor Biology, vol. 26, no. 1, pp. 1-8, 2005.

[17] F. Cianchi, A. Palomba, L. Messerini et al., "Tumor angiogenesis in lymph node-negative rectal cancer: correlation with clinicopathological parameters and prognosis," Annals of Surgical Oncology, vol. 9, no. 1, pp. 20-26, 2002.

[18] Y. S. Hong, H. J. Cho, S. Y. Kim et al., "Carbonic anhydrase 9 is a predictive marker of survival benefit from lower dose of bevacizumab in patients with previously treated metastatic colorectal cancer," BMC cancer, vol. 9, article 246, 2009.

[19] J. Giralt, B. Navalpotro, E. Hermosilla et al., "Prognostic significance of vascular endothelial growth factor and cyclooxygenase- 2 in patients with rectal cancer treated with preoperative radiotherapy," Oncology, vol. 71, no. 5-6, pp. 312319, 2006.

[20] S. Miyagawa, S. Miwa, J. Soeda, A. Kobayashi, and S. Kawasaki, "Morphometric analysis of liver macrophages in patients with colorectal liver metastasis," Clinical and Experimental Metastasis, vol. 19, no. 2, pp. 119-125, 2002.

[21] Y.-C. Chung, Y.-C. Hou, C.-N. Chang, and T.-H. Hseu, "Expression and prognostic significance of angiopoietin in colorectal carcinoma," Journal of Surgical Oncology, vol. 94, no. 7, pp. 631638, 2006.

[22] R. Rajaganeshan, D. G. Jayne, H. Z. Malik et al., "Biological characteristics and behaviour of putatively curatively resected colorectal liver metastases," European Journal of Surgical Oncology, vol. 34, no. 4, pp. 439-444, 2008.

[23] A. Nanashima, K. Shibata, T. Nakayama et al., "Clinical significance of microvessel count in patients with metastatic liver cancer originating from colorectal carcinoma," Annals of Surgical Oncology, vol. 16, no. 8, pp. 2130-2137, 2009. 
[24] Y. Toiyama, Y. Inoue, S. Saigusa et al., "Gene expression profiles of epidermal growth factor receptor, vascular endothelial growth factor and hypoxia-inducible factor- 1 with special reference to local responsiveness to neoadjuvant chemoradiotherapy and disease recurrence after rectal cancer surgery," Clinical Oncology, vol. 22, no. 4, pp. 272-280, 2010.

[25] S.-C. Wei, J.-T. Liang, P.-N. Tsao, F.-J. Hsieh, S.-C. Yu, and J.$\mathrm{M}$. Wong, "Preoperative serum placenta growth factor level is a prognostic biomarker in colorectal cancer," Diseases of the Colon and Rectum, vol. 52, no. 9, pp. 1630-1636, 2009.

[26] K. A. Kwon, S. H. Kim, S. Y. Oh et al., "Clinical significance of preoperative serum vascular endothelial growth factor, interleukin-6, and C-reactive protein level in colorectal cancer," BMC Cancer, vol. 10, article 203, 2010.

[27] T. Miyazaki, N. Okada, K. Ishibashi et al., "Clinical significance of plasma level of vascular endothelial growth factor-C in patients with colorectal cancer," Japanese Journal of Clinical Oncology, vol. 38, no. 12, pp. 839-843, 2008.

[28] S.-I. Ishigami, S. Arii, M. Furutani et al., "Predictive value of vascular endothelial growth factor (VEGF) in metastasis and prognosis of human colorectal cancer," British Journal of Cancer, vol. 78, no. 10, pp. 1379-1384, 1998.

[29] D. Cao, M. Hou, Y.-S. Guan, M. Jiang, Y. Yang, and H.-F. Gou, "Expression of HIF-lalpha and VEGF in colorectal cancer: association with clinical outcomes and prognostic implications," BMC Cancer, vol. 9, article 432, 2009.

[30] P. Ferroni, A. Spila, F. Martini et al., "Prognostic value of vascular endothelial growth factor tumor tissue content of colorectal cancer," Oncology, vol. 69, no. 2, pp. 145-153, 2005.

[31] J.-F. Liang, H.-K. Wang, H. Xiao et al., "Relationship and prognostic significance of SPARC and VEGF protein expression in colon cancer," Journal of Experimental and Clinical Cancer Research, vol. 29, no. 1, article 71, 2010.

[32] E. Kaio, S. Tanaka, Y. Kitadai et al., "Clinical significance of angiogenic factor expression at the deepest invasive site of advanced colorectal carcinoma," Oncology, vol. 64, no. 1, pp. 6173, 2003.

[33] A. A. Khorana, C. K. Ryan, C. Cox, S. Eberly, and D. M. Sahasrabudhe, "Vascular endothelial growth factor, CD68, and epidermal growth factor receptor expression and survival in patients with stage II and stage III colon carcinoma: a role for the host response in prognosis," Cancer, vol. 97, no. 4, pp. 960968, 2003.

[34] M. Tamura, M. Oda, Y. Tsunezuka, I. Matsumoto, K. Kawakami, and G. Watanabe, "Vascular endothelial growth factor expression in metastatic pulmonary tumor from colorectal carcinoma: utility as a prognostic factor," Journal of Thoracic and Cardiovascular Surgery, vol. 128, no. 4, pp. 517-522, 2004.

[35] J. D. White, P. W. Hewett, D. Kosuge et al., "Vascular endothelial growth factor-D expression is an independent prognostic marker for survival in colorectal carcinoma," Cancer Research, vol. 62, no. 6, pp. 1669-1675, 2002.

[36] M. Kojima, A. Shiokawa, N. Ohike et al., "Clinical significance of nuclear morphometry at the invasive front of T1 colorectal cancer and relation to expression of VEGF-A and VEGF-C," Oncology, vol. 68, no. 2-3, pp. 230-238, 2005.

[37] A. A. Alabi, A. Suppiah, L. A. Madden, J. R. Monson, and J. Greenman, "Preoperative serum vascular endothelial growth factor-A is a marker for subsequent recurrence in colorectal cancer patients," Diseases of the Colon and Rectum, vol. 52, no. 5, pp. 993-999, 2009.
[38] Z.-G. Zhou, X.-J. Wu, L.-R. Li et al., "A multivariate analysis of prognostic determinants for stages II and III colorectal cancer in 141 patients," Chinese Medical Journal, vol. 124, no. 14, pp. 2132-2135, 2011.

[39] K. Zafirellis, G. Agrogiannis, A. Zachaki, K. Gravani, A. Karameris, and C. Kombouras, "Prognostic significance of VEGF expression evaluated by quantitative immunohistochemical analysis in colorectal cancer," Journal of Surgical Research, vol. 147, no. 1, pp. 99-107, 2008.

[40] J. Chen, Q. Li, C. Wang, J. Wu, and G. Zhao, "Prognostic significance of c-erbb-2 and vascular endothelial growth factor in colorectal liver metastases," Annals of Surgical Oncology, vol. 17, no. 6, pp. 1555-1563, 2010.

[41] C. Zhang, L. Hao, L. Wang et al., "Elevated IGFIR expression regulating VEGF and VEGF-C predicts lymph node metastasis in human colorectal cancer," BMC Cancer, vol. 10, article 184, 2010.

[42] M. Lin, H.-Z. Lin, S.-P. Ma, P. Ji, D. Xie, and J.-X. Yu, "Vascular endothelial growth factor-A and -C: expression and correlations with lymphatic metastasis and prognosis in colorectal cancer," Medical Oncology, vol. 28, no. 1, pp. 151-158, 2011.

[43] H. Amaya, N. Tanigawa, C. Lu et al., "Association of vascular endothelial growth factor expression with tumor angiogenesis, survival and thymidine phosphorylase/platelet-derived endothelial cell growth factor expression in human colorectal cancer," Cancer Letters, vol. 119, no. 2, pp. 227-235, 1997.

[44] Y. Harada, Y. Ogata, and K. Shirouzu, "Expression of vascular endothelial growth factor and its receptor KDR (kinase domaincontaining receptor)/Flk-1 (fetal liver kinase-1) as prognostic factors in human colorectal cancer," International Journal of Clinical Oncology, vol. 6, no. 5, pp. 221-228, 2001.

[45] J.-C. Lee, N.-H. Chow, S.-T. Wang, and S.-M. Huang, "Prognostic value of vascular endothelial growth factor expression in colorectal cancer patients," European Journal of Cancer, vol. 36, no. 6, pp. 748-753, 2000.

[46] S. Zheng, M.-Y. Han, Z.-X. Xiao, J.-P. Peng, and Q. Dong, "Clinical significance of vascular endothelial growth factor expression and neovascularization in colorectal carcinoma," World Journal of Gastroenterology, vol. 9, no. 6, pp. 1227-1230, 2003.

[47] A.-M. Ochs, L. Wong, V. Kakani et al., "Expression of vascular endothelial growth factor and HER2/neu in stage II colon cancer and correlation with survival," Clinical Colorectal Cancer, vol. 4, no. 4, pp. 262-267, 2004.

[48] S. M. Kang, K. Maeda, N. Onoda et al., "Combined analysis of p53 and vascular endothelial growth factor expression in colorectal carcinoma for determination of tumor vascularity and liver metastasis," International Journal of Cancer, vol. 74, pp. 502-507, 1997.

[49] G. E. Theodoropoulos, A. C. Lazaris, V. E. Theodoropoulos et al., "Hypoxia, angiogenesis and apoptosis markers in locally advanced rectal cancer," International Journal of Colorectal Disease, vol. 21, no. 3, pp. 248-257, 2006.

[50] S. Shinji, Z. Naito, T. Ishiwata et al., "Neuroendocrine cell differentiation of poorly differentiated colorectal adenocarcinoma correlates with liver metastasis," International Journal of Oncology, vol. 29, no. 2, pp. 357-364, 2006.

[51] E. Kaio, S. Tanaka, S. Oka et al., "Clinical significance of thrombospondin-1 expression in relation to vascular endothelial growth factor and interleukin-10 expression at the deepest invasive tumor site of advanced colorectal carcinoma," International Journal of Oncology, vol. 23, no. 4, pp. 901-911, 2003. 
[52] A. M. Jubb, H. I. Hurwitz, W. Bai et al., "Impact of vascular endothelial growth factor-A expression, thrombospondin-2 expression, and microvessel density on the treatment effect of bevacizumab in metastatic colorectal cancer," Journal of Clinical Oncology, vol. 24, no. 2, pp. 217-227, 2006.

[53] L. R. Moreira, A. A. Schenka, P. Latuf-Filho et al., "Immunohistochemical analysis of vascular density and area in colorectal carcinoma using different markers and comparison with clinicopathologic prognostic factors," Tumor Biology, vol. 32, no. 3, pp. 527-534, 2011.

[54] M. Gulubova and T. Vlaykova, "Prognostic significance of mast cell number and microvascular density for the survival of patients with primary colorectal cancer," Journal of Gastroenterology and Hepatology, vol. 24, no. 7, pp. 1265-1275, 2009.

[55] P. B. Vermeulen, G. G. van den Eynden, P. Huget et al., "Prospective study of intratumoral microvessel density, p53 expression and survival in colorectal cancer," British Journal of Cancer, vol. 79, no. 2, pp. 316-322, 1999.

[56] T. Sternfeld, H. D. Foss, M. Kruschewski, and N. Runkel, “The prognostic significance of tumor vascularization in patients with localized colorectal cancer," International Journal of Colorectal Disease, vol. 14, no. 6, pp. 272-276, 1999.

[57] N. Tanigawa, H. Amaya, M. Matsumura et al., "Tumor anglogenesis and mode of metastasis in patients with colorectal cancer," Cancer Research, vol. 57, no. 6, pp. 1043-1046, 1997.

[58] A. A. Romani, A. F. Borghetti, P. del Rio, M. Sianesi, and P. Soliani, "The risk of developing metastatic disease in colorectal cancer is related to CD105-positive vessel count," Journal of Surgical Oncology, vol. 93, no. 6, pp. 446-455, 2006.

[59] Y. Takebayashi, S. I. Akiyama, S. Akiba et al., "Clinicopathologic and prognostic significance of an angiogenic factor, thymidine phosphorylase, in human colorectal carcinoma," Journal of the National Cancer Institute, vol. 88, pp. 1110-1117, 1996.

[60] T. Hasebe, S. Sasaki, M. Sugitoh, M. Ono, N. Saitoh, and A. Ochiai, "Proliferative activities of tumor stromal cells play important roles in tumor thickness and progression of T3 ulcerative-type colorectal cancer," Virchows Archiv, vol. 442, no. 6, pp. 569-576, 2003.

[61] S. Svagzdys, V. Lesauskaite, D. Pavalkis, I. Nedzelskiene, D. Pranys, and A. Tamelis, "Microvessel density as new prognostic marker after radiotherapy in rectal cancer," BMC Cancer, vol. 9, article 95, 2009.

[62] G. Yan, X.-Y. Zhou, S.-J. Cai, G.-H. Zhang, J.-J. Peng, and X. $\mathrm{Du}$, "Lymphangiogenic and angiogenic microvessel density in human primary sporadic colorectal carcinoma," World Journal of Gastroenterology, vol. 14, no. 1, pp. 101-107, 2008.

[63] Y. Yonenaga, A. Mori, H. Onodera et al., "Absence of smooth muscle actin-positive pericyte coverage of tumor vessels correlates with hematogenous metastasis and prognosis of colorectal cancer patients," Oncology, vol. 69, no. 2, pp. 159-166, 2005.

[64] C. Tarta, C. R. Teixeira, S. Tanaka, K. Haruma, C. ChieleNeto, and V. D. da Silva, "Angiogenesis in advanced colorectal adenocarcinoma with special reference to tumoral invasion," Arquivos de Gastroenterologia, vol. 39, no. 1, pp. 32-38, 2002.

[65] N. Pietra, L. Sarli, P. Caruana et al., "Is tumour angiogenesis a prognostic factor in patients with colorectal cancer and no involved nodes?" European Journal of Surgery, vol. 166, no. 7, pp. 552-556, 2000.

[66] H. J. Choi, M. S. Hyun, G. J. Jung, S. S. Kim, and S. H. Hong, "Tumor angiogenesis as a prognostic predictor in colorectal carcinoma with special reference to mode of metastasis and recurrence," Oncology, vol. 55, no. 6, pp. 575-581, 1998.
[67] S. Tomisaki, S. Ohno, Y. Ichiyoshi, H. Kuwano, Y. Maehara, and K. Sugimachi, "Microvessel quantification and its possible relation with liver metastasis in colorectal cancer," Cancer, vol. 77, pp. 1722-1728, 1996.

[68] N. Minagawa, Y. Nakayama, K. Hirata et al., "Correlation of plasma level and immunohistochemical expression of vascular endothelial growth factor in patients with advanced colorectal cancer," Anticancer Research, vol. 22, no. 5, pp. 2957-2964, 2002.

[69] Y. Takebayashi, S. I. Akiyama, K. Yamada, S. Akiba, and T. Aikou, "Angiogenesis as an unfavorable prognostic factor in human colorectal carcinoma," Cancer, vol. 78, pp. 226-231, 1996.

[70] N. Sharifi, K. Ghaffarzadegan, H. Ayatollahi, M. T. Shakeri, M. H. Sadeghian, and J. B. Azari, "Evaluation of angiogenesis in colorectal carcinoma by CD34 immunohistochemistry method and its correlation with clinicopathologic parameters," Acta Medica Iranica, vol. 47, no. 3, pp. 161-164, 2009.

[71] S. Rasheed, A. L. Harris, P. P. Tekkis et al., "Assessment of microvessel density and carbonic anhydrase-9 (CA-9) expression in rectal cancer," Pathology Research and Practice, vol. 205, no. 1, pp. 1-9, 2009.

[72] N. Teranishi, Z. Naito, T. Ishiwata et al., "Identification of neovasculature using nestin in colorectal cancer," International Journal of Oncology, vol. 30, no. 3, pp. 593-603, 2007.

[73] A. Nanashima, M. Ito, I. Sekine et al., "Significance of angiogenic factors in liver metastatic tumors originating from colorectal cancers," Digestive Diseases and Sciences, vol. 43, no. 12, pp. 2634-2640, 1998.

[74] M. F. Acikalin, Ü. Öner, I. Topçu, B. Yaşar, H. Kiper, and E. Çolak, "Tumour angiogenesis and mast cell density in the prognostic assessment of colorectal carcinomas," Digestive and Liver Disease, vol. 37, no. 3, pp. 162-169, 2005.

[75] P. Zhan, J. Wang, X.-J. Lv et al., "Prognostic value of vascular endothelial growth factor expression in patients with lung cancer: a systematic review with meta-analysis," Journal of Thoracic Oncology, vol. 4, no. 9, pp. 1094-1103, 2009.

[76] R. A. Smith, J. Tang, C. Tudur-Smith, J. P. Neoptolemos, and P. Ghaneh, "Meta-analysis of immunohistochemical prognostic markers in resected pancreatic cancer," British Journal of Cancer, vol. 104, no. 9, pp. 1440-1451, 2011.

[77] S. J. Schoenleber, D. M. Kurtz, J. A. Talwalkar, L. R. Roberts, and G. J. Gores, "Prognostic role of vascular endothelial growth factor in hepatocellular carcinoma: systematic review and metaanalysis," British Journal of Cancer, vol. 100, no. 9, pp. 1385-1392, 2009.

[78] J. Chen, T. Li, Y. Wu et al., "Prognostic significance of vascular endothelial growth factor expression in gastric carcinoma: a meta-analysis," Journal of Cancer Research and Clinical Oncology, vol. 137, no. 12, pp. 1799-1812, 2011.

[79] N. Ferrara, "Vascular endothelial growth factor: basic science and clinical progress," Endocrine Reviews, vol. 25, no. 4, pp. 581611, 2004.

[80] L. G. Presta, H. Chen, S. J. O’Connor et al., "Humanization of an anti-vascular endothelial growth factor monoclonal antibody for the therapy of solid tumors and other disorders," Cancer Research, vol. 57, no. 20, pp. 4593-4599, 1997.

[81] H. Hurwitz, "Integrating the anti-VEGF-a humanized monoclonal antibody bevacizumab with chemotherapy in advanced colorectal cancer," Clinical Colorectal Cancer, vol. 4, supplement 2, pp. S62-S68, 2004.

[82] L. Saltz, "Management of metastatic colorectal cancer: the impact of clinical trials reported at ASCO 2007," Community Oncology, vol. 5, no. 1, pp. 37-42, 2008. 
[83] F. F. Kabbinavar, J. Hambleton, R. D. Mass, H. I. Hurwitz, E. Bergsland, and S. Sarkar, "Combined analysis of efficacy: the addition of bevacizumab to fluorouracil/leucovorin improves survival for patients with metastatic colorectal cancer," Journal of Clinical Oncology, vol. 23, no. 16, pp. 3706-3712, 2005.

[84] Y. Cao, A. Tan, F. Gao, L. Liu, C. Liao, and Z. Mo, "A metaanalysis of randomized controlled trials comparing chemotherapy plus bevacizumab with chemotherapy alone in metastatic colorectal cancer," International Journal of Colorectal Disease, vol. 24 , no. 6 , pp. 677-685, 2009. 


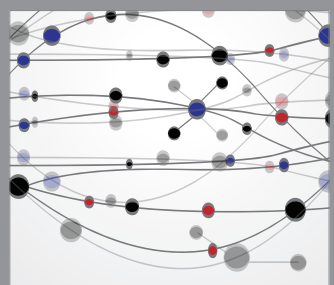

The Scientific World Journal
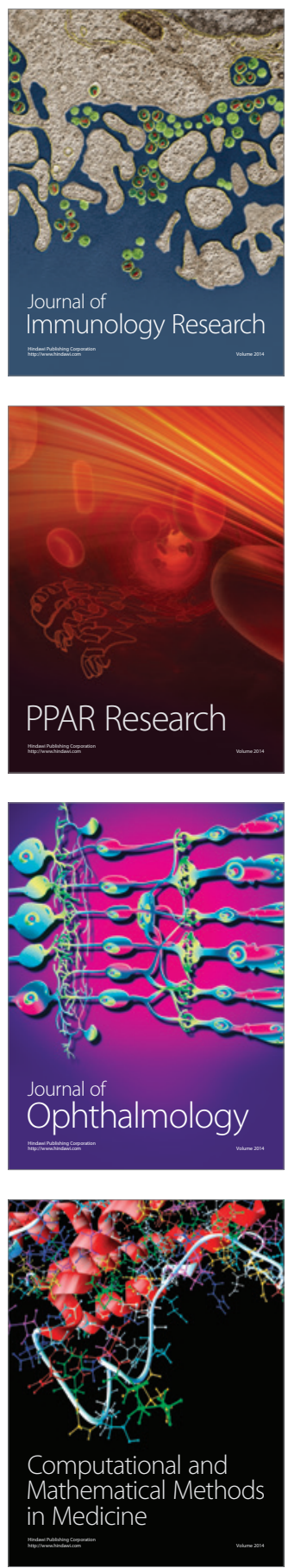

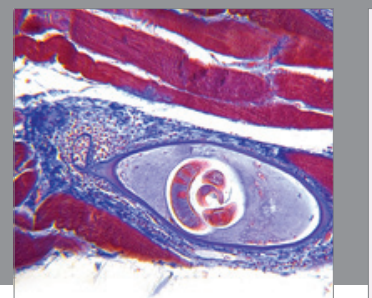

Gastroenterology

Research and Practice
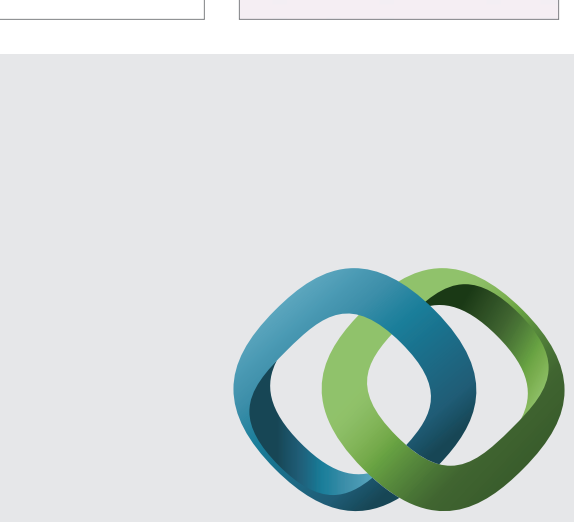

\section{Hindawi}

Submit your manuscripts at

http://www.hindawi.com
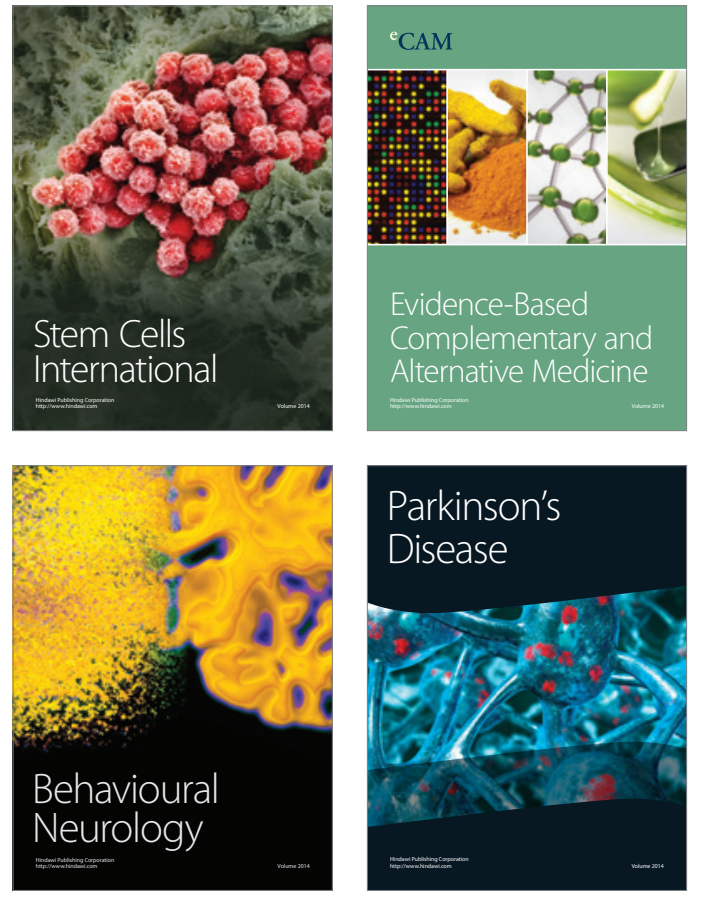
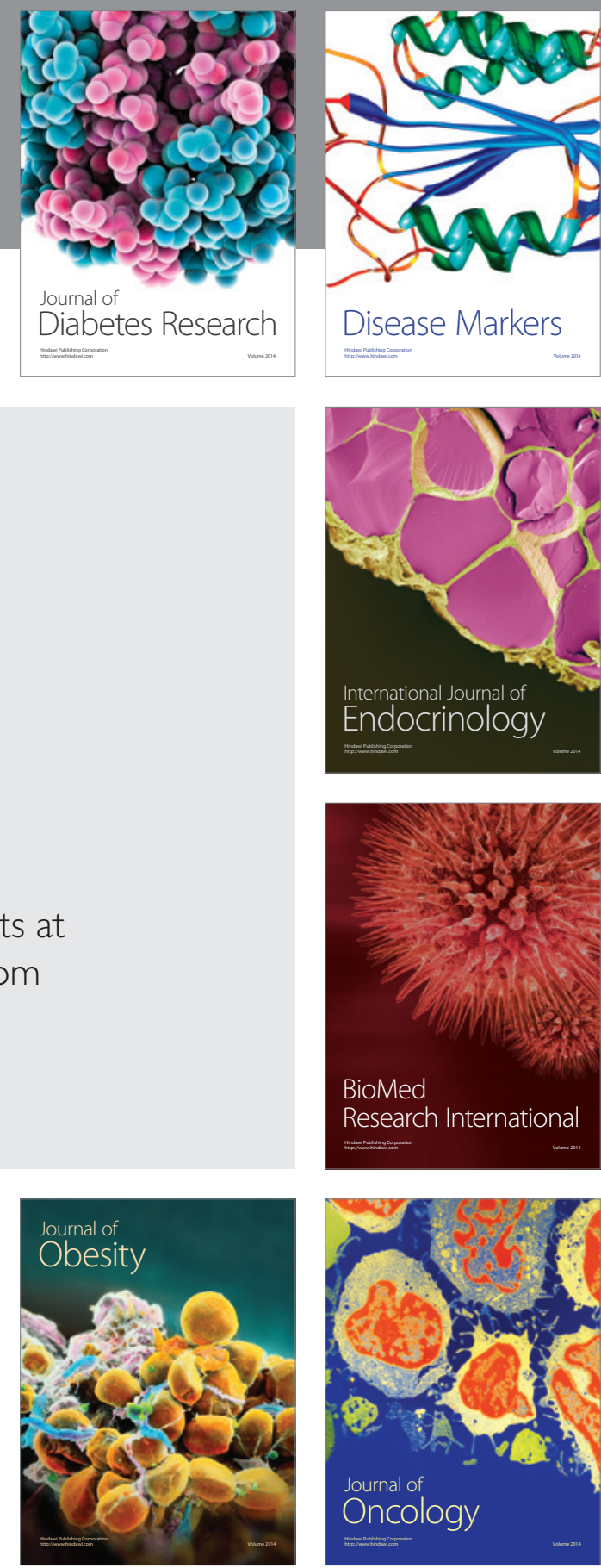

Disease Markers
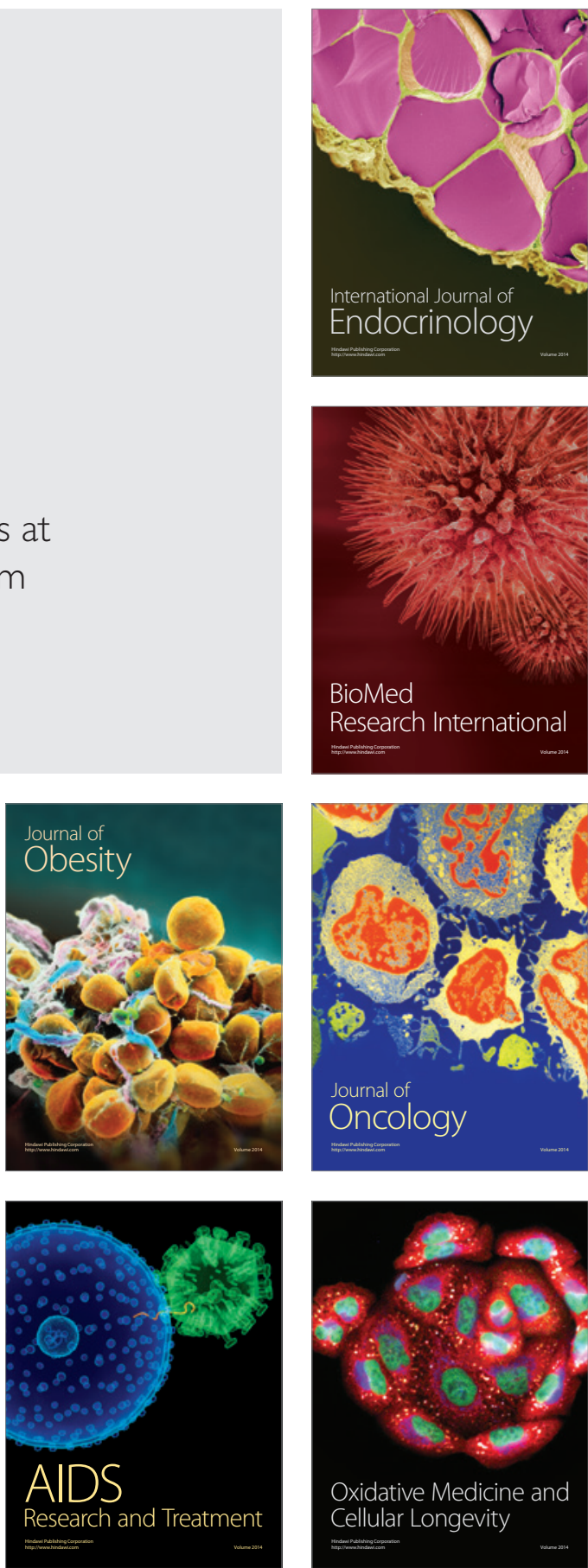